\title{
HLA-A Mismatch Count
}

National Cancer Institute

\section{Source}

National Cancer Institute. HLA-A Mismatch Count. NCI Thesaurus. Code C128956.

The determination of the number of mismatch between the study subject and his or her donor for the human leukocyte antigen, class I, group A (HLA-A). 